\title{
Microstructural Evolution in Transition-metal-oxide Cathode Materials for Lithium-Ion Batteries
}

\author{
Dean J. Miller ${ }^{1}$, Huaping Sheng ${ }^{1}$, Lifen Wang ${ }^{1}$, J.G. Wen ${ }^{1}$, Jun $\mathrm{Lu}^{2}$, Khalil Amine ${ }^{2}$, James A. Gilbert ${ }^{2}$, \\ Matilda Klett $^{2}$, Javier Bareno ${ }^{2}$, Victor A. Maroni ${ }^{2}$, Daniel P. Abraham ${ }^{2}$ \\ 1. Electron Microscopy Center - Center for Nanoscale Materials, Argonne National Laboratory, USA \\ 2. Chemical Sciences and Engineering Division, Argonne National Laboratory, USA
}

Layered transition metal oxides are promising materials for high-energy lithium-ion battery cathodes. These materials offer high capacity and rate capability, good safety, and relatively low cost compared to many alternative materials [1]. Mixed oxides such as $\mathrm{NCA}\left(\mathrm{LiNi}_{0.8} \mathrm{Co}_{0.15} \mathrm{Al}_{0.05} \mathrm{O}_{2}\right)$ exhibit high capacity but suffer from a significant capacity fade with cycling [2]. The composition of alternatives such as NMC ( $\left.\mathrm{LiNi}_{1-\mathrm{x}-\mathrm{y}} \mathrm{Mn}_{\mathrm{x}} \mathrm{Co}_{\mathrm{y}} \mathrm{O}_{2}\right)$ can be tuned to show less capacity fade, but generally at the cost of lower capacity. Consequently, there is great interest in cycling these materials to higher potentials for more energy, but then cycling performance decreases.

The capacity fade in these materials has been attributed to a variety of factors including reactions with the electrolyte, phase transformations and the formation of insulating phases, and Li loss to SEI layers. It is likely all of these mechanisms contribute to some degree, and cycled cells that have been disassembled, cleaned, and reassembled with a new source of lithium can show a significant recovery of capacity. However, the capacity fade is also generally associated with an impedance rise at the positive electrode that is not recovered, suggesting permanent damage to the cathode [2].

There is growing evidence that mechanical degradation of the cathode plays a significant role in this impedance rise and corresponding capacity fade [3]. In previous work, we used conventional and operando electron microscopy to reveal grain boundary separations and cracking in NCA during electrochemical cycling that we believe is a major factor to capacity fade [4]. In this work, focused on NMC and "gradient" cathode materials to see if mechanical degradation was an important factor in their performance. Gradient cathode materials have an overall NMC composition, but with a gradient in composition to achieve an Ni-rich core to provide high capacity and an Mn-rich periphery to minimize detrimental interaction with the electrolyte. [5]

For NMC, cycling over a wider voltage window is suggested to increase capacity, but leads to significant capacity fade. Electron microscopy reveals that mechanical degradation - grain boundary separations and cracking - is significant in cathode particles extracted from coin cells, even after only 100 cycles, as show in Fig. 1. This is consistent with a model in which separations lead to a loss of grain-to-grain connectivity within particles could contribute to a degradation of performance and loss of capacity, especially by causing an increase in impedance, as in observed in these materials.

Gradient material based on NMC compositions are of significant interest because they tend to show much less capacity fade on cycling compared to their ungraded counterparts. While this is often ascribed to reduced chemical interactions due to the Mn-rich periphery, we find a significant difference in the cracking behavior of these materials that is linked to their microstructure and that we feel also plays a role in their improved performance. Fig. 2 shows a cross-section image of a particle harvested from a coin cell. After 50 cycles, this particle shows little cracking, and the cracks that are observed are radial following the grain structure and morphology of the particle. The smaller grain size is more resistant to fracture [3], and the radial grain structure leads to radial cracks that do not lead to isolated 
grains that can reduce capacity. However, compositional profiles measured by EDS in TEM (Fig. 2) confirm the overall gradient morphology, but also reveal evidence for gradients within individual grains. Thus we cannot exclude the additional contribution of reduced surface reactions on the new surfaces that are exposed when cracks do form, which is the topic of further studies.

\section{References:}

[1] M.S. Whittingham, Chem. Rev. 2004, 104, 4271-4301].

[2] Y. Li, et al., Journal of The Electrochemical Society, 160 (5) A3006-A3019 (2013).

[3] W.H. Woodford, et al., Energy Environ. Sci., 2012,5, 8014-8024.

[4] D.J. Miller, et al., Adv. Energy Mater. 3 (8) 1098-1103 (2013)

[5] Y.-K. Sun, et al., Adv. Funct. Mater. 20, 485-491 (2010)

[6] Research in the Center for Nanoscale Materials is supported by the U. S. Department of Energy, Office of Science under Contract No. DE-AC02-06CH11357
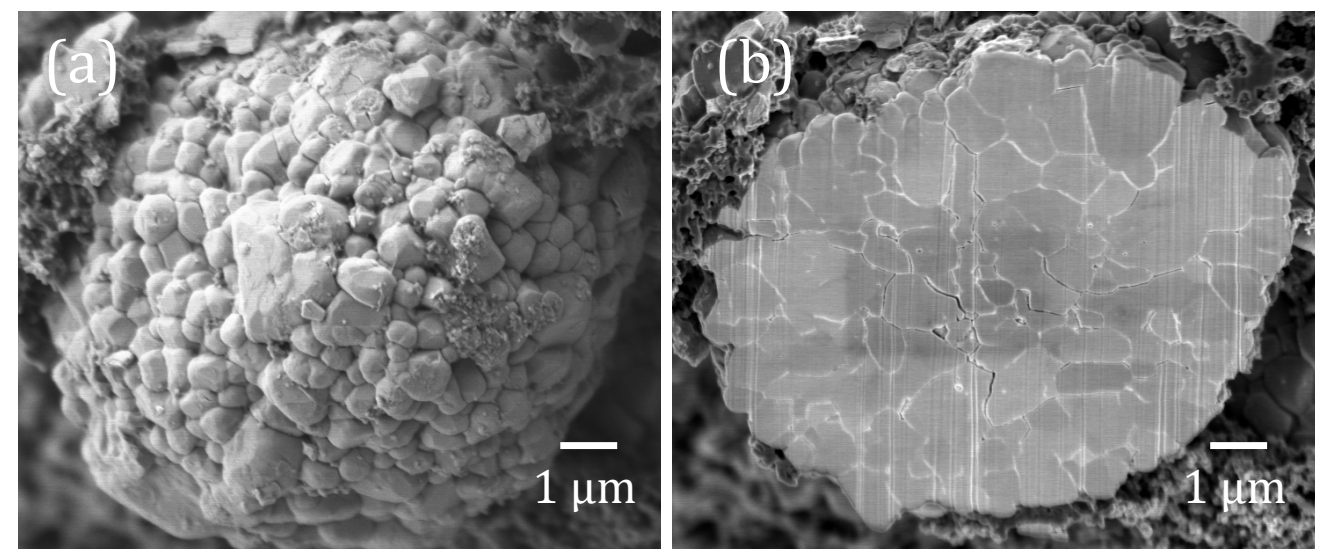

Figure 1. SEM images of the (a) surface and (b) cross-section of an $\mathrm{Li}_{1.03}\left(\mathrm{Ni}_{0.5} \mathrm{Mn}_{0.3} \mathrm{Co}_{0.2}\right)_{0.97} \mathrm{O}_{2}$ particle harvested from a coin cell that had been cycled 100 times. The cross-sectional image in (b) reveals separations at nearly all of the grain boundaries.
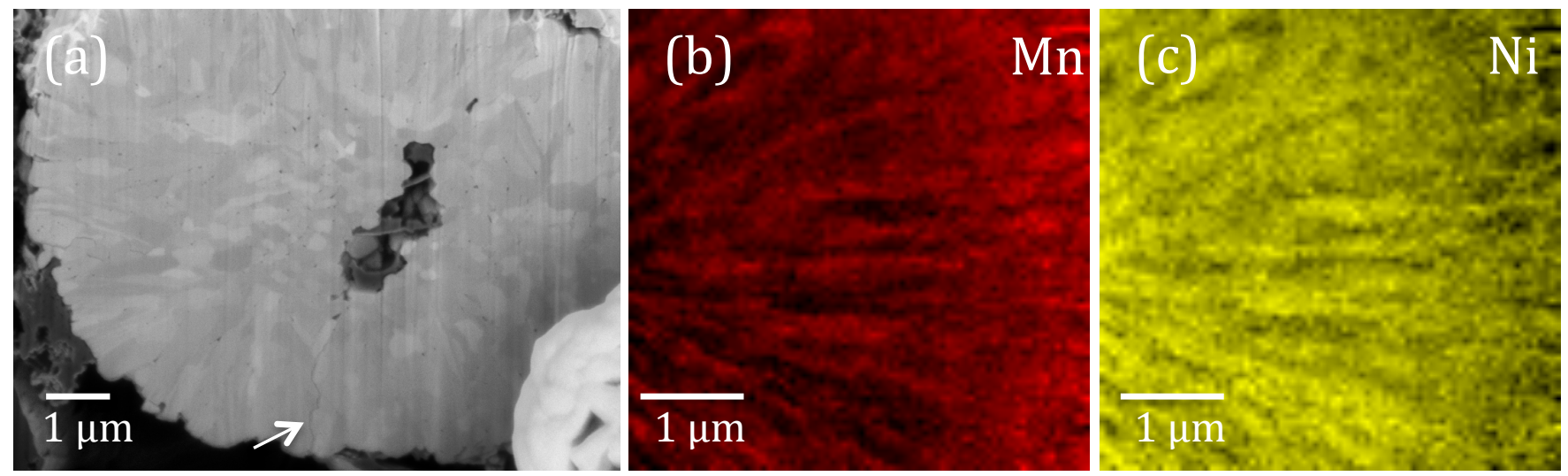

Figure 2. (a) SEM cross-sectional image of an NMC gradient particle harvested from a coin cell that had been cycled 50 times. There are only a very few cracks, one of which is indicated by an arrow. The core-shell-like morphology with a radial grain structure is characteristic of these gradient materials. EDS spectral mapping from TEM on a different particle for (b) Mn and (c) Ni. 